\title{
Quel rapport entre passé et présent dans les manuels scolaires d'histoire en France et en Allemagne?
}

How do history textbooks relate to the past and the present in France and in Germany?

\section{Patricia von Münchow}

\section{OpenEdition Journals}

Édition électronique

URL : http://journals.openedition.org/aad/2429

DOI : 10.4000/aad.2429

ISSN : $1565-8961$

Éditeur

Université de Tel-Aviv

Référence électronique

Patricia von Münchow, « Quel rapport entre passé et présent dans les manuels scolaires d'histoire en France et en Allemagne? », Argumentation et Analyse du Discours [En ligne], 19 | 2017, mis en ligne le 15 octobre 2017, consulté le 23 septembre 2019. URL : http://journals.openedition.org/aad/2429 ; DOI : 10.4000/aad.2429

Ce document a été généré automatiquement le 23 septembre 2019.

\section{cc) (†) $\odot$}

Argumentation \& analyse du discours est mis à disposition selon les termes de la licence Creative Commons Attribution - Pas d'Utilisation Commerciale - Pas de Modification 4.0 International. 


\section{Quel rapport entre passé et présent dans les manuels scolaires d'histoire en France et en Allemagne?}

How do history textbooks relate to the past and the present in France and in

Germany?

Patricia von Münchow

\section{Introduction}

1 Cet article présente une partie des résultats d'une analyse du discours contrastive récente de manuels d'histoire en France et en Allemagne, et plus précisément des chapitres portant sur la Première Guerre Mondiale. Il s'agit de montrer, à l'aide d'une série d'entrées d'analyse fondées sur différentes opérations discursives, quelles représentations se font les auteurs des manuels étudiés de la Première Guerre Mondiale en tant que telle, mais aussi de ce conflit en tant qu'objet d'apprentissage : que doit apprendre l'élève, à l'aide du manuel ? On s'intéresse plus particulièrement au rapport entre le passé abordé dans les manuels et le présent de la situation d'enseignement/ apprentissage. Après avoir évoqué les rapports entre analyse du discours et manuels scolaires, on rappelle brièvement le cadre théorico-méthodologique de l'analyse du discours contrastive et on explique les modalités de construction du corpus. On passe alors en revue un certain nombre d'entrées d'analyse permettant de mieux comprendre quel rapport entre passé et présent s'établit dans les différents manuels avant de conclure sur les représentations construites par l'ensemble de ces procédés.

\section{Analyse du discours et manuel scolaire}

2 Le manuel scolaire continue à occuper une place centrale dans le champ éducatif (Ott 2015 : 20) : à l'ère de l'information surabondante, il exerce plus que jamais une fonction 
d'orientation (Dreesen 2015 : 79). Ainsi est-il régulièrement étudié d'un point de vue linguistique, discursif ou didactique, la majorité des travaux de ces dernières années relevant, selon Ott (2015: 21), de la linguistique anglophone et en particulier de la Critical Discourse Analysis. Parmi les études récentes et qui se placent, de près ou de loin, dans la perspective d'analyse du discours qu'on adopte ici, on peut notamment mentionner les travaux que mènent Dreesen et Judkowiak (2011, par exemple), dans le cadre de ce qu'ils appellent « linguistique du discours contrastive »'. L'ouvrage dirigé par Christophe et Schwedes (2015) sur le traitement de la Première Guerre Mondiale dans les manuels d'une série de pays différents est également à signaler, même si les auteurs se placent plutôt dans le cadre de la «Kulturwissenschaft» («sciences de la culture ») et livrent des descriptions linguistiques certes fines, mais qui reposent sur des catégories analytiques non spécialisées, et sont toujours subordonnées à un questionnement qui n'est pas discursif à proprement parler.

3 Pour Christophe et Schwedes (2015a: 9), le manuel scolaire et en particulier le manuel d'histoire «regroupe comme dans un verre brûlant» la ou les "culturelles mémorielles » de la communauté pour laquelle il a été fabriqué ${ }^{2}$. Comme le montrait déjà Halbwachs (1997 [1950]), la culture mémorielle est toujours façonnée à la fois par les événements (de différentes époques) du passé et par les besoins du présent, ces derniers pouvant tout à fait être multiples et opposés les uns aux autres (Christophe et Schwedes 2015b : 23). Les auteures s'étonnent (2015b: 17) de ce que le manuel ne soit pas plus souvent lu comme un "texte mémoriel» alors qu'il permet de comprendre comment on se voyait soi-même et comment on voyait le monde à différents moments et à différents endroits. C'est ce type de lecture qu'on propose ici en interrogeant, à l'aide des outils de l'analyse du discours contrastive, le rapport entre passé et présent construit dans les manuels.

\section{Cadre théorico-méthodologique}

4 L'analyse du discours contrastive est une approche qui relève des sciences du langage, au carrefour de l'analyse du discours française, de la linguistique textuelle et des approches contrastives ou "transculturelles $»^{3}$. Son objet est la comparaison de différentes cultures discursives, notion qui recouvre la construction/manifestation discursive des représentations sociales circulant dans une communauté sur les objets sociaux, d'une part, et sur les discours à tenir sur ces objets sociaux, d'autre part. C'est en tant que linguiste, en partant d'opérations discursives, que je m'efforce d'atteindre ces représentations sociales. En l'occurrence, on tente de comprendre quel positionnement dans le temps construisent les auteurs des manuels d'histoire à travers un certain nombre de procédés discursifs qu'on détaille ci-dessous.

\section{Corpus}

5 Le corpus étudié consiste en quatre manuels d'histoire français et sept manuels allemands, tous récents et en usage au moment de l'établissement du corpus ${ }^{4}$ (2012-2014) :

- Arias, S. \& E. Chaudron (dir.). 2012. Histoire Géographie $3^{e}$ (Paris : Belin) [Belin]

- Azzouz, R. et M.-L. Gache (dir.). 2012. Histoire Géographie $3^{e}$ (Paris : Magnard) [Magnard]

- Hazard-Tourillon A.-M. \& A. Fellahi (dir.). 2012. Histoire Géographie $3^{e}$ (Paris : Nathan) [Nathan] 
- Ivernel, M. \& B. Villemagne (dir.). 2012. Histoire Géographie $3^{e}$ (Paris : Hatier) [Hatier]

- Ebeling, H. \& W. Birkenfeld (dir.). 2013. Die Reise in die Vergangenheit. Nordrhein-Westfalen 2 (Braunschweig: Westermann) [Reise]

- Lendzian, H.-J. \& W. Mattes (dir.). 2005. Zeiten und Menschen 3 (Paderborn : Schöningh)

- Oomen, H.-G. (dir.). 2013. Geschichte Hessen. Entdecken und Verstehen 3. Von der Französischen Revolution bis zum Ersten Weltkrieg (Berlin : Cornelsen) [Entdecken]

- Regenhardt, H.-O. \& C. Tatsch (dir.). 2009. Forum Geschichte. Ausgabe Hessen. Band 4: Vom Ersten Weltkrieg bis heute (Berlin : Cornelsen) [Forum]

- Sauer, M. (dir.). 2011. Geschichte und Geschehen 5 (Stuttgart/Leipzig : Klett) [Geschichte]

- Simianer, N. (dir.). 2006. Von...bis. Geschichtsbuch für Realschulen (Braunschweig/Paderborn/ Darmstadt : Schöningh) [Von...bis]

- Christoffer, S. et al. 2012. Mitmischen 2 (Stuttgart/Leipzig : Klett) [Mitmischen]

Les manuels allemands étudiés sont plus nombreux que les manuels français pour que plusieurs Länder, de même que différentes filières du secondaire (Gymnasium, Realschule, Hauptschule, Gesamtschule), soient représentés par le corpus. Cela étant, on ne pourra aborder ici, par manque de place, les différences observées entre les manuels allemands selon le Land ou la filière pour lesquels ils sont prévus. Les ouvrages français s'adressent tous à un public du niveau de la troisième alors que les manuels allemands sont censés pouvoir être utilisés de façon plus flexible entre l'équivalent de la quatrième et de la seconde (8. Klasse, 9. Klasse, 10. Klasse), selon les Länder.

\section{Résultats}

7 Dans les pages qui suivent on présentera les résultats de l'étude contrastive effectuée en examinant les titres des manuels et les temps linguistiques employés au fil des textes, en passant en revue différents types de description et de discours rapporté, en mettant en évidence les comparaisons entre passé et présent et en montrant de quelle manière l'élève apprend à produire des discours adaptés à l'époque de la Première Guerre Mondiale.

\subsection{Les titres des manuels}

Les manuels d'histoire affichent dès la couverture le rapport qu'ils privilégient entre présent et passé. Les manuels français s'appelant tous Histoire Géographie $3^{e}$, l'objet annoncé est une discipline scolaire, qui se situe a priori dans le présent. Les manuels allemands ont tous des titres différents dont certains sont assez explicites quant au positionnement dans le temps. Ainsi Die Reise in die Vergangenheit (Le voyage dans le passé) renvoie-t-il à l'idée de déplacement du présent vers le passé. Le titre Geschichte und Geschehen n'est pas aisé à traduire : « Histoire et événement »? « Temps révolu et temps continu »? Dans tous les cas, un rapport entre passé et présent est annoncé. Enfin, Mitmischen (qu'on pourrait traduire par « Participer » ou « S'en mêler ») semble inscrire au cœur des événements l'action de l'acteur qu'est probablement l'élève. L'articulation de cette annonce au genre discursif du manuel d'histoire peut faire naître l'idée qu'il s'agit pour l'élève de participer à l'histoire, ce qui, une nouvelle fois, établit potentiellement un rapport entre présent et passé, même si l'on ne comprend pas tout de suite de quelle manière l'élève pourrait bien intervenir dans l'histoire révolue. 


\subsection{Le positionnement dans le temps linguistique}

La deuxième entrée analytique qu'on mobilise consiste en l'examen des temps au sens linguistique à proprement parler. On observe d'abord que le temps de base dans les manuels d'histoire français est le présent, alors que c'est le prétérit qui est le plus fréquent dans les manuels allemands. On peut supposer une parenté privilégiée du manuel français avec le discours didactique ou encore encyclopédique alors que le manuel allemand se rapprocherait plutôt du discours historiographique ou d'autres genres de la mémoire, généralement marqués par les temps du "récit ", d'après Weinrich (1985 [1964] : 20).

Mais davantage que sur ce qui semble finalement être des conventions génériques différentes d'un pays à l'autre il faut, à mon sens, se concentrer sur la manière dont ces temps de base s'articulent à d'autres temps ou à d'autres marqueurs temporels, aspectuels, évidentiels, etc. Ainsi dans l'extrait suivant, on observe "l'irruption » du passé composé au milieu d'une série de présents («Des Poilus ont décrit [...]» au dernier paragraphe) :

(1) Après l'assassinat de François-Ferdinand d'Autriche en juin 1914 à Sarajevo,

l'engrenage des alliances entraîne l'Europe dans une guerre qui mobilise 70 millions de soldats venus de tous les continents [...].

D'août à décembre 1914, c'est la guerre de mouvement [...] les états-majors lancent des offensives très meurtrières.

Aucun belligérant n'ayant remporté de victoires décisives, les armées s'enterrent dans des tranchées. [...]

Des Poilus ont décrit l'horreur des combats et des conditions de vie dans les tranchées [...]. Ils font l'expérience de la mort et de la peur, du froid, de la boue, des poux, des rats et des difficultés de ravitaillement. En 1917, les soldats français et allemands se mutinent (Magnard : 32$)^{5}$.

11 Selon Weinrich (1985 [1964]: 58), qui, dans le domaine de la Sprecherperspektive ("perspective de locution») distingue Vorausschau ("perspective en avant») et Rückschau (" perspective en arrière »), le présent n'exprime aucune perspective. C'est le passé composé qui introduit une perspective en arrière à calculer à partir de la situation d'énonciation. Il établit donc un rapport entre notre époque et l'époque révolue. Dans le contexte dans lequel il apparaît dans l'extrait ci-dessus, on constate que ce qui entre en rapport avec nous, ce qui nous concerne, dans la logique de la construction temporelle du manuel, ce ne sont pas les événements de la Première Guerre Mondiale, mais leur représentation, leur catégorisation verbale, ce qu'il en reste à l'écrit, ce qui est "devenu source». On peut rapprocher cette observation de celle portant sur les titres des manuels français, qui réfèrent à la discipline scolaire et donc au passé tel qu'il est étudié et non tel qu'il s'est déroulé.

12 L'absence de perspective sur les événements de la guerre en tant que tels est encore plus manifeste dans des énoncés ne comportant pas de verbes et donc pas de temps grammatical du tout. Dans le passage suivant, tiré d'un texte intitulé «Les grandes phases de la guerre ", on peut distinguer cinq phrases dont trois sont averbales :

(2) 1914 : guerre de mouvement

- Août 1914 : les Allemands envahissent la France.

- 6-13 septembre : contre-offensive de l'armée française, lors de la bataille de la

Marne. Les Allemands reculent. Environ 500000 morts (Nathan : 43).

13 On a preque affaire ici à une énumération, c'est-à-dire non pas à un récit, mais à un «degré zéro de la procédure descriptive " (Adam 1992: 81). Ainsi semble-t-il que ce 
qu'on présente à l'élève est moins ce qui s'est passé que ce qu'il doit retenir, en vue non pas d'une entrée en relation avec le passé, mais d'une réussite dans des épreuves d'évaluation.

Quant aux manuels allemands, ils sont marqués, sur le plan de la Sprechhaltung (" attitude de locution »), par le temps du « récit» (toujours au sens de Weinrich) qu'est le prétérit. Ce dernier n'introduisant pas plus de perspective dans le récit que ne le fait le présent dans le cadre du " commentaire ", il faut, là aussi, étudier d'autres temps ou modes ainsi que l'emploi des déictiques pour se faire une meilleure idée du rapport entre présent et passé dans les manuels en question. Dans l'extrait suivant, le déictique jetzt («maintenant ») indique le déplacement du locuteur dans le passé, au moment du déclenchement de la guerre. Le futur dans le passé à valeur prospective («dauern würde », fr. « durerait ») a le même effet :

(3) Die Stimmung in Europa war im Sommer 1914 von Kriegsbegeisterung und Siegeszuversicht geprägt. Menschen, die sich für den Frieden einsetzten, wurden jetzt oft als Feiglinge oder „Vaterlandsverräter” beschimpft. Fast jeder glaubte an die Kriegsschuld der Gegner und war bereit, sein vermeintlich bedrohtes Land zu verteidigen. Kaum jemand konnte sich vorstellen, dass der Krieg länger als ein paar Wochen dauern würde. Diese Meinungen wurden durch Propaganda unterstützt (Reise : 271).

[L'ambiance en Europe pendant l'été de 1914 était marquée par l'enthousiasme pour la guerre et la certitude de vaincre. Les personnes qui militaient pour la paix se faisaient maintenant traiter de lâches ou de "traîtres à la patrie ". Presque tous croyaient que les ennemis étaient responsables de la guerre et étaient prêts à défendre leur pays qu'ils pensaient menacé. Presque personne ne pouvait imaginer que la guerre durerait plus de quelques semaines. Ces opinions étaient soutenues par la propagande.]

Dans l'extrait suivant, le titre au présent - et donc dans l'attitude de locution du commentaire - est suivi d'un discours direct dont l'énoncé rapportant s'incrit dans le récit par l'intermédiaire du prétérit, ce qui est également le cas de l'énoncé qui clôt l'extrait :

(4) Europa im Juli 1914 - wie ein „Weltbrand” entsteht

„In Europa gehen die Lichter aus. Wir werden es nicht mehr erleben, wenn sie wieder angehen." Mit diesen Worten beschrieb der englische Außenminister Grey am Abend des 4. August 1914 die Zukunft Europas [...]. Wie konnte es dazu kommen - und sollte er Recht behalten? (Geschichte : 68)

[L'Europe en juillet 1914 - comment naît un « incendie mondial »

« Les lumières s'éteignent en Europe. Nous ne les verrons plus se rallumer de notre vivant." C'est par ces mots que le ministre des affaires étrangères anglais Grey décrivit l'avenir de l'Europe le soir du 4 août 1914 [...]. Comment est-ce que cela avait pu arriver - et devait-il avoir raison ?]

L'énoncé rapporté au discours direct, aux temps du présent et du futur, permet de nouveau de se projeter en 1914. Ensuite, la dernière phrase de l'extrait ouvre d'abord, par l'intermédiaire du sémantisme du verbe (rendu en traduction par le plus-queparfait), une perspective en arrière à partir de 1914, puis il introduit une perspective en avant - également à partir de 1914 - grâce à l'emploi prospectif du verbe modal " sollen ». Rebotier (2011) parle à ce sujet d'un "présent futural » alors que Confais (2002 : 19) évoque la combinaison d'une valeur temporelle et d'une valeur modale : le rôle de "sollen " prospectif «semble être moins de positionner un événement dans le temps que de suggérer une "préparation" de l'événement ". Dans tous les cas, on a affaire à un point de départ qui s'établit au 4 août 1914 et qui donne lieu à une 
perspective en arrière aussi bien qu'en avant. Ainsi s'établissent des liens entre différents moments ou périodes dans le passé. Quelle peut être la motivation pour ce tissage de liens complexe ? L'extrait suivant, d'une source cette fois, permet de mieux le comprendre :

(5) Der US-Außenminister Lansing schrieb am 8. Mai 1919:

Die Friedensbedingungen erschienen unsagbar hart und demütigend. [...] Hass und Erbitterung, wenn nicht Verzweiflung müssen die Folgen derartiger Bestimmungen sein. Wir haben einen Friedensvertrag, aber er wird keinen dauernden Frieden bringen (Reise : 282). [Le ministre des affaires étrangères américain Lansing écrivit le 8 mai 1919:

Les conditions de la paix semblaient incroyablement dures et humiliantes. [...] La haine et l'amertume, sinon le désespoir seront très probablement des conséquences de telles dispositions. Nous avons un traité de paix, mais il n'apportera pas une paix durable.]

On retrouve ici, à partir du point de départ que constitue le 8 mai 1919, une perspective en avant, prise en charge par un locuteur rapporté américain se positionnant comme arbitre de l'Europe, qui reflète l'importance attribuée dans les manuels allemands à la question de la responsabilité dans le déclenchement de la guerre, d'une part, et à celle de l'évaluation du traité de Versailles, d'autre part, le tout dans la perspective des événements qui ont suivi à partir de 1933 et qui relèguent la Première Guerre Mondiale à une place tout à fait secondaire. On entrevoit donc ici l'importance prépondérante du nazisme et de la Seconde Guerre Mondiale, période qui structure fortement le discours public allemand, exerçant à son tour une pression considérable - et sans égale du côté français - sur celui des manuels d'histoire. La perspective en avant à partir de la Première Guerre Mondiale permet d'établir une continuité entre les événements de 1914-1919 et ceux d'à partir de 1933. On remarquera cependant que les auteurs du manuel préfèrent faire parler une source étrangère pour établir ce rapport, sans doute parce qu'il n'est pas possible - c'est-à-dire discursivement convenable - pour un auteur allemand d'expliquer, ne serait-ce que partiellement, l'avènement du nazisme par une erreur de jugement des Alliés lors de l'élaboration du Traité de Versailles.

\subsection{Descriptions détaillées}

18 Un autre aspect à examiner au sujet du rapport avec le passé qui s'établit dans les manuels est la présence ou non d'un certain type de description. Dans l'extrait suivant d'un manuel allemand, on constate que la vie au front est décrite de façon détaillée. Sont mentionnés jusqu'aux éléments sur lesquels peuvent peser des tabous discursifs (la puanteur, les morceaux de cadavres) :

(6) Wochenlang mussten sie [die Soldaten] bei Regen und Kälte, unter feindlichem Beschuss und voller Todesangst in stinkenden Gruben, umgeben von Leichenteilen und Ungeziefer, aushalten (Reise : 272).

[Pendant des semaines, ils [les soldats] devaient supporter de rester, dans la pluie et le froid, sous les tirs de l'ennemi et remplis d'angoisse de mort, dans des fossés puants, entourés de cadavres déchiquetés et de cafards.]

19 Dans l'extrait qui suit, la description des effets de l'artillerie comporte une série d'intensifications produites par la quantification et les verbes métaphoriques :

(7) Die Gewalt der Explosionen zerfetzte in Minuten ganze Wälder, stampfte Betonbunker zusammen und zerriss Hunderttausende von Menschen (Entdecken: 148).

[La violence des explosions déchiquetait en quelques minutes des forêts entières, pilonnait des bunkers en béton et dépeçait des centaines de milliers de personnes.] 

invalide de guerre ayant perdu les deux bras et mangeant avec des prothèses très rudimentaires (Reise : 277). Dans l'ensemble, il s'agit sans doute de donner au lecteurélève l'impression « d'y être ».

Dans les manuels français, les descriptions (sur)détaillées et intensifiées apparaissent exclusivement dans les sources et non dans les textes de présentation des auteurs. Les sources en question sont souvent des journaux de guerre et des lettres du front :

(8) «[...] Les Allemands, avec une ténacité inouie, avec une violence sans égale, attaquent nos lignes qu'ils martèlent et rongent [...]. Nos poilus héroïques tiennent bon, malgré les déluges d'acier, de liquides enflammés, de gaz asphyxiants. [...] » D'après le Journal de guerre du docteur Marcel POISOT (Belin : 30)

(9) «[...] tous mes camarades sont tombés morts ou blessés aux mains des Boches qui nous ont fait souffrir les mille horreurs, liquides enflammés, gaz asphyxiants, attaques [...]. » Lettre de Georges Gallois, $221^{\mathrm{e}}$ régiment d'infanterie, Paroles de poilus, Librio, 1998 (Hatier : 40).

Les auteurs donnent la parole aux personnes ayant vécu la guerre, les mettent en scène, tout en restant eux-mêmes à distance de ces situations et des personnes qui les ont vécues. Les sources sont aussi l'occasion de faire apparaître des descriptions accablantes du comportement des Allemands, que les auteurs s'abstiennent systématiquement de livrer dans leurs textes de présentation.

Enfin, certaines descriptions dans les manuels allemands comportent des détails à première vue inutiles :

(10) Am 11. November 1918 wurde der Waffenstillstand in einem Eisenbahnwaggon im Wald von Compiegne unterzeichnet (Von... bis : 101).

[Le 11 novembre 1918 l'armistice fut signée dans un wagon de chemin de fer dans la forêt de Compiègne.]

24 En effet, le wagon et la forêt de Compiègne ne contribuent pas à une meilleure compréhension des (micro- ou macro-)événements. Mais ces détails ont néanmoins une fonction: ils participent à la transmission d'une certaine mémoire collective. Ils existent aussi dans des récits français de la guerre, mais ne figurent pas dans les manuels. Les auteurs français ne souhaitent peut-être pas inscrire leurs élèves dans ce type de mémoire.

\subsection{Discours rapporté}

L'emploi du discours rapporté dans les manuels est révélateur, lui aussi, du rapport au passé dans lequel s'inscrivent les auteurs. Dans les ouvrages français, le type de discours rapporté principalement actualisé dans les textes de présentation des auteurs est le discours indirect par l'intermédiaire duquel sont représentés des discours constituant des actes politiques (von Münchow 2016b). Il s'agit de faire état d'événements consistant en des discours, en accentuant l'opération de catégorisation de l'acte de parole aux dépens de la paraphrase de ce qui a été dit (Authier-Revuz 2012 : 159). Le discours direct, quant à lui, est rare dans ces manuels. C'est comme si l'on voulait insister sur le fait qu'une médiation pédagogique doit toujours s'interposer entre le discours «d'époque » et l'élève, ce dernier devant retenir quels actes ont été produits et non quels mots ont été prononcés. Les auteurs allemands, quant à eux, mobilisent bien davantage le discours direct. Cela peut se faire dans un but de dramatisation, comme dans cette source :

Argumentation et Analyse du Discours, 19 | 2017 
(11) Ein Soldat schrieb kurz vor seinem Tod vor Verdun:

Liebe Eltern! Ich liege auf dem Schlachtfeld mit Bauchschuss. Ich glaube, ich muss sterben.

Dank euch, ihr lieben Eltern! Gott befohlen, Hans. (Reise : 275)

[Un soldat écrivit peu de temps avant sa mort à Verdun :

Chers parents, Je suis allongé sur le champ de bataille avec une balle dans le ventre. Je crois

que je vais mourir. Merci à vous, chers parents ! Adieu, Hans.]

Aucune consigne exigeant un effort de généralisation ne suit ce témoignage individuel et on peut supposer que l'élève est censé développer une attitude empathique envers le soldat à qui on donne ici la parole, voire s'identifier à lui pour mieux comprendre ce que signifie la guerre. Mais le plus souvent les locuteurs dont les énoncés sont rapportés au discours direct ne sont pas des anonymes, mais des dirigeants politiques. La citation suivante de l'Empereur Guillaume II figure dans le manuel en caractères gothiques, sans doute pour intensifier encore l'effet de mimétisme que produit le discours direct :

(12) „Aus dem Deutschen Reich ist ein Weltreich geworden.”

(Kaiser Wilhelm II. am 18. Januar 1896 auf der Feier des 25. Jahrestages der

Reichsgründung) (Von...bis : 80)

[ «e Reich allemand est devenu un Reich mondial. »

(Empereur Guillaume II le 18 janvier 1896 lors de la célébration des 25 ans de la

fondation du Reich)]

L'élève doit, autant que possible, opérer un déplacement temporel et "se sentir être " en 1896 pour entendre l'Empereur parler.

En dehors des types de discours rapporté dans lesquels le discours autre est l'objet de l'affirmation (Authier-Revuz 2012: 158), on relève aussi une série de modalisations autonymiques d'emprunt (MAE) $)^{6}$ dont le «locuteur rapporté » est «la masse " anonyme. Là encore, les occurrences dans les manuels français sont plutôt rares et concernent essentiellement l'expérience traumatisante de la guerre :

(13) La « génération du feu » sort traumatisée de la guerre [...] (Hatier : 44).

(14) « L'enfer de Verdun » (Hatier : 40)

Il s'agit dans les deux cas de désignations toujours en emploi et tout à fait acceptables aujourd'hui, sans doute parce que les combattants de toutes les nations pourraient être le référent de la première et que la deuxième pouvait être utilisée dans tous les pays pour référer à la bataille de Verdun. Ces deux MAE contribuent donc à la construction de la perspective européenne contemporaine qui caractérise plus généralement les manuels français (von Münchow 2016a). «L'enfer de Verdun» apparaît d'ailleurs également dans les manuels allemands :

(15) Die „Hölle von Verdun” wurde zum Inbegriff dieses Materialkrieges (Forum :

20).

[« L'enfer de Verdun » est devenu le symbole de cette guerre de positions.]

30 La MAE suivante, en revanche, correspond bien à un discours de 1919 (et des années qui ont suivi), plus du tout acceptable aujourd'hui, et, qui plus est, à un discours allemand de 1919:

(16) Viele sahen in den Regierungsmitgliedern, die den Vertrag von Versailles unterzeichnet hatten, die wahren Schuldigen an diesem „Schandfrieden” (Forum : 283).

[Beaucoup de gens voyaient les membres du gouvernement qui avaient signé le traité de Versailles comme étant les vrais coupables de cette " paix de la honte ».]

31 On a ici affaire à un marquage discret de la MAE par l'intermédiaire de guillemets, marquage qui permet aux élèves de prendre connaissance d'un certain discours du 
passé et de se familiariser avec un patrimoine discursif en "l'entendant", tout en comprenant que les termes en question ne peuvent pas être utilisés aujourd'hui de façon non autonymique : dans l'Allemagne $\mathrm{du} 21^{\mathrm{e}}$ siècle, on risque la marginalisation si l'on parle publiquement d'une « paix de la honte » pour référer au Traité de Versailles.

Dans certains cas, cependant, la MAE n'est pas marquée du tout et son identification devient entièrement interprétative :

(17) Deutschland über alle(s)

Es ist nicht richtig, wenn Menschen meinen, ihr eigenes Volk sei besser als andere Völker. Doch schon vor hundert Jahren glaubten das viele (Mitmischen : 280).

[Deutschland über alle(s) ${ }^{7}$

Ce n'est pas bien de penser que son propre peuple est meilleur que les autres. Pourtant il y a cent ans déjà nombreux sont ceux qui le croyaient.]

On a ici affaire à une allusion au début du Deutschlandlied. Celle-ci est non marquée mais facile à reconnaître - en tout cas pour des adultes - étant donné la notoriété de ce vers devenu une formule, qui a changé de sens plusieurs fois au cours de son histoire. N'est retenu ici que le sens pangermaniste, déjà en circulation au moment de la Première Guerre Mondiale, mais qui ne s'est réellement imposé que plus tard. L'auteur porte une appréciation sur la formule et en même temps sur l'énoncé qui suit («wenn Menschen meinen, ihr eigenes Volk sei besser als andere Völker », fr. " quand les gens pensent que leur propre peuple est meilleur que les autres »). Cette formule est donc en même temps exposée et dépréciée: l'élève doit connaître les discours de l'époque de la Première Guerre Mondiale tels qu'ils étaient, mais il doit également apprendre à les évaluer. Dans les consignes suivantes, la déconstruction d'une autre formule va plus loin encore :

(18) Auf dem Bild M1 [«Gedenkblatt zum deutsch-französischen Krieg 1870/1871»] wird dargestellt, was damals viele Menschen unter der "Größe Deutschlands“ verstanden:

a) Was gehörte alles zur "nationalen Größe Deutschlands“?

b) Welche Personen waren demnach "große Deutsche“? [l'image montre des empereurs, des chanceliers et des princes]

c) Welche Rolle spielten Kriege und Schlachten, wenn es darum ging, die „nationale Größe Deutschlands" aufzuzeigen?

d) Welche Ereignisse und Personen fehlen auf dem Bild, auf die die Deutschen stolz sein konnten?

[...] Nenne drei deutsche Männer und Frauen, die in deinen Augen „große Deutsche“ sind. Begründe kurz deine Meinung (Mitmischen : 280-281).

[Sur l'image M1 [«Feuillet commémoratif de la guerre franco-allemande 1870/1871 »] est représenté ce que beaucoup de gens entendaient alors par la «grandeur de l'Allemagne»:

a) À quoi renvoyait la « grandeur nationale de l'Allemagne »?

b) Par conséquent, quelles personnes étaient de «grands Allemands»? [l'image montre des empereurs, des chanceliers et des princes]

c) Quel rôle jouaient les guerres et les batailles lorsqu'il s'agissait de témoigner de la " grandeur nationale de l'Allemagne »?

d) Quels sont les événements et les personnes dont les Allemands pouvaient être fiers et qui manquent sur l'image?

[...] Donne les noms de trois hommes et femmes allemands qui sont de "grands Allemands » à tes yeux. Justifie rapidement ton opinion.]

L'élève doit ici réinvestir d'un nouveau sens les formules, en adoptant d'abord un point de vue de la seconde moitié du 19e siècle, puis un point de vue du 21e siècle. Dans l'ensemble, on essaie de faire comprendre une époque à l'élève en lui faisant 
" entendre ", voire "sentir ", à force de répétition, les mots du passé. Un certain patrimoine discursif allemand est ainsi transmis, que l'élève doit non seulement entendre et assimiler, mais aussi déconstruire, voire réinvestir d'un autre sens possible, mais non attesté à l'époque étudiée. Il doit aussi réfléchir au nouveau sens que pourrait prendre une formule aujourd'hui. C'est comme si on ne pouvait faire autrement que de bâtir sur le passé, y compris, en l'occurrence, sur les mots de ce passé.

\subsection{Comparaisons entre passé et présent}

Les comparaisons entre passé et présent permettent, elles aussi, de tisser un lien entre l'époque de la Première Guerre Mondiale (au sens large) et les années 2010. Elles sont exclusivement présentes dans les manuels d'histoire allemands. Dans la consigne suivante, on demande à l'élève d'opérer d'abord une comparaison entre deux discours de l'époque sous examen, puis de comparer les positionnements au sujet de la colonisation datant de cette époque au discours actuel sur la globalisation :

(19) Stelle die Ausführungen Bebels [contre la colonisation] und Dernburgs [pour la colonisation] gegenüber [...]. Vergleiche mit Argumenten für und gegen die „Globalisierung" heute (Geschichte : 56).

[Confronte les explications de Bebel [contre la colonisation] à celles de Dernburg [pour la colonisation] [...]. Compare à des arguments pour et contre la «globalisation » aujourd'hui.]

La comparaison avec l'époque actuelle peut aussi porter sur des réalités autres que des discours. Dans le cadre de la section «Familien im Krieg» («Des familles pendant la guerre ») du manuel Mitmischen (p. 296-297), les textes des auteurs et les sources détaillent la situation dramatique de la population : pénurie de nourriture, inquiétude pour les soldats au front, nouvelles de leur mort. Dans l'une des sources (M3, p. 297), tiré du journal intime d'une inconnue (Anna Kohns) vivant à Bonn, on trouve une liste détaillée des quantités de nourriture auxquelles chacun avait droit dans le cadre du rationnement alimentaire. S'y rapporte la consigne suivante :

(20) Wiege $100-150 \mathrm{~g}$ Fleisch und $30 \mathrm{~g}$ Fett ab.

a) Wie lange kommst du mit dieser Ration aus?

b) Zeige M3 [Lebensmittelliste] deinen Eltern. Frage sie, wie hoch der wöchentliche Verbrauch der genannten Lebensmittel bei euch ist (Mitmischen : 297).

[Pèse 100 à $150 \mathrm{~g}$ de viande et $30 \mathrm{~g}$ de graisse.

a) Combien de temps tu tiens avec cette ration?

b) Montre M3 [liste d'aliments] à tes parents. Demande-leur quelle quantité hebdomadaire des aliments en question vous consommez chez vous.]

On a l'impression d'avoir affaire à la mise en œuvre d'une pédagogie par l'action ici. Selon Friedl, Christophe et Schwedes (2015: 98), l'enseignement orienté vers l'action est en effet l'une des formes de mise en œuvre en didactique de l'histoire de ce que Bergmann (1979, 2000) appelle la «multiperspectivité» (Multiperspektivität). L'établissement explicite d'un rapport avec le présent (Gegenwartsbezug) fait également partie des mots d'ordre des courants contemporains de la didactique de l'histoire en Allemagne (Friedl, Christophe et Schwedes 2015 : 97-98). Il ne suffit pas que l'élève sache de façon abstraite quelles étaient précisément les quantités d'aliments dont on devait se contenter pendant la guerre, il doit les voir, les regarder en face, les confronter à sa propre réalité pour mieux prendre conscience de la pénurie que subissaient les familles. En France, après avoir marqué certains manuels des années 1990, ce rapport passé-présent ne semble plus être d'actualité. Ainsi Baquès (2007 : 146) 
observe-t-elle (dans les manuels destinés aux lycéens), un retour en arrière après l'échec du Magnard de seconde de 1996, considéré comme "présentiste » en raison de la confrontation systématique de débats actuels à des problèmes passés, confrontation non reprise en 2001 et réintroduite seulement de façon timide et « distanciée » en 2005.

\subsection{L'élève comme producteur d'un discours du passé}

Enfin, l'élève allemand est parfois appelé à produire lui-même un « discours du passé ». À propos d'une affiche appelant les femmes allemandes à contribuer à la victoire par leur travail dans l'industrie de l'armement, la consigne est la suivante :

(21) Drei Frauen stehen vor dem Plakat und unterhalten sich darüber. Die Erste hat fünf Kinder zu ernähren. Die Zweite hat einen Feldpostbrief von ihrem Mann erhalten, in dem er die schlechte Ausrüstung der Soldaten beklagt. Die Dritte hat gerade ihren Sohn an der Front verloren. Spielt diese Szene nach [Geschichte : 83]. [Trois femmes conversent devant l'affiche. La première a cinq enfants à nourrir. La deuxième a reçu une lettre $d u$ front de la part de son mari dans laquelle il se plaint $\mathrm{du}$ mauvais équipement des soldats. La troisième vient de perdre son fils au front. Jouez cette scène.]

Le déplacement dans le temps que l'élève doit opérer de façon récurrente est parfois associé à un déplacement dans l'espace. Il s'agit de se mettre à la place de citoyens de l'époque de la Première Guerre Mondiale dans un autre pays que l'Allemagne et de produire « leur » discours :

(22) Schreibt als Brite einen Brief an Admiral von Tirpitz, in dem ihr auf die Gefahren der deutschen Flottenpolitik hinweist (Entdecken : 141).

[Écrivez en tant que Britannique une lettre à l'Amiral von Tirpitz, dans laquelle vous indiquez les dangers de la politique de la flotte allemande.]

(23) Beurteilt den Versailler Vertrag aus der Sicht eines damaligen Franzosen und eines damaligen Deutschen (Entdecken : 161).

[Évaluez le traité de Versailles dans la perspective d'un Français de l'époque et d'un Allemand de l'époque.]

Ces consignes s'incrivent sans doute également dans «la multiperspectivité », qui implique pour l'élève un déplacement spatial aussi bien que temporel. Enfin, ce déplacement peut aussi s'opérer à l'intérieur du champ des positionnements possibles à l'époque de la Première Guerre Mondiale au sein même de la communauté allemande, comme le montre l'extrait suivant de la section «War der Friede noch zu retten?» («Pouvait-on encore sauver la paix ?») :

(24) Versetzt euch in einen Deutschen, der im August 1914 gegen den Krieg ist. Sammelt für ihn Argumente, mit denen er seine Mitbürger überzeugen

könnte (Entdecken : 145).

[Mettez-vous à la place d'un Allemand qui est contre la guerre en août 1914.

Rassemblez pour lui des arguments avec lesquels il pourrait convaincre ses concitoyens.]

L'exercice consiste ici à se mettre à la place de ceux qui n'ont pas fait l'Histoire et de produire à leur place un discours qui aurait peut-être permis à l'Histoire de se dérouler autrement. C'est en quelque sorte un discours doublement virtuel qu'il s'agit de construire : l'élève s'imagine non seulement comme étant au milieu des événements de 1914, mais, qui plus est, au milieu de ceux qui n'ont pas eu lieu, mais auraient pu se produire. Cette situation n'est pas envisagée d'un point de vue contemporain, mais d'un point de départ dans le passé : le subjonctif dans l'énoncé allemand exprime l'irréel du présent et non du passé. 

un impératif didactique récent souligné entre autres par Friedl, Christophe et Schwedes (2015 : 97-98) : mise en évidence de la « controversité » (Kontroversität) d'un événement - autrement dit, du fait que même à l'intérieur d'une communauté, il n'est pas nécessairement interprété de la même façon (voir aussi Pandel 2005: 34) - et promotion de la compréhension de l'Autre. La production d'un tel discours du passé n'est pas demandée à l'élève français, et Baquès (2007: 125) fait remarquer que la "perspective critique », qu'on peut rapprocher de la "controversité », apparue dans les manuels français dans les années 1980, en a disparu à la fin des années 1990. L'élève est sans doute censé garder une certaine distance vis à vis du passé en général et de ses discours en particulier.

\section{Conclusion}

Cette étude a permis de montrer que le rapport qui s'établit entre le présent de la situation d'enseignement/apprentissage et le passé qui est l'objet de l'enseignement/ apprentissage de l'Histoire n'est pas le même dans les manuels français et allemands. La perspective contemporaine dans les manuels français s'oppose au va-et-vient entre présent et passé qu'on constate dans les ouvrages allemands. Alors que l'attitude qui s'impose par rapport au passé dans les livres français est marquée par une forte distance, les manuels allemands incitent l'élève à pratiquer une sorte d'empathie critique. Le passé doit y être "ressenti»; il est en quelque sorte transposé dans le présent et virtuellement transformé en ce qu'il aurait pu ou dû être, alors qu'il est plutôt exposé dans les sources reproduites dans les ouvrages français, sans intervention apparente des auteurs. Ainsi présent et passé sont-ils mis en relation dans les manuels allemands, alors qu'ils restent sans lien dans les ouvrages français. Dans ces derniers, les discours du passé sont évités, peut-être parce qu'ils seraient dangereux et susceptibles de ranimer des antagonismes désormais surmontés entre pays européens. Dans les ouvrages d'outre-Rhin, en revanche, les discours du passé semblent inévitables : ils sont utilisés, déconstruits et remotivés ou "transmotivés » pour faire entrer l'élève en interaction avec le passé alors que les manuels français le maintiennent « dans son époque ».

Dans l'ensemble on a affaire, dans les ouvrages français, à la construction d'un élève européen qui ne peut ni ne doit, en tant que tel, s'identifier à un citoyen de l'époque de la Première Guerre Mondiale. Cela semble participer de la finalité récente, en didactique de l'histoire, de "faire de l'Europe un nouveau socle d'"appartenance" " (Lautier et Allieu-Mary 2008). Il s'agit dans cette perspective de faire acquérir à l'élève un discours sur les événements vécus par les citoyens du début du $20^{\mathrm{e}}$ siècle et non pas de l'amener à se mettre à leur place : il doit apprendre ce qu'il convient de dire, au $21^{\mathrm{e}}$ siècle, au sujet de la guerre et non pas ce que disaient ou ressentaient les ressortissants des pays belligérants en 1914 ou en 1918. Conformément à l'objectif didactique de la «multiperspectivité » déjà évoqué (Bergmann 1979, 2000), l'élève allemand, en revanche, est incité à s'identifier à un citoyen de l'époque de la Première Guerre Mondiale, à prendre connaissance du discours de ce citoyen, voire à le reproduire et à comprendre de l'intérieur ce qui s'est passé à cette époque.

La perspective « vers l'avenir » qu'on constate dans les manuels allemands lorsqu'il est question de la Première Guerre Mondiale indique sans doute que le « point nodal » de

Argumentation et Analyse du Discours, 19 | 2017 
l'histoire allemande est la Deuxième Guerre Mondiale ${ }^{9}$, omniprésente dans le chapitre sur la guerre précédente. L'un des facteurs intervenant dans cet état de fait est l'Histoire elle-même, bien entendu. Le nazisme et la Deuxième Guerre Mondiale ont donné lieu à des crimes inédits et ont eu des conséquences bien plus lourdes pour l'Allemagne que la Première Guerre Mondiale sur une série de plans, mais surtout en termes de responsabilité morale. Il n'est pas étonnant dès lors que cette époque ait fini par devenir - à partir des années 1970 - omniprésente dans le discours public et pédagogique, et que ce qui précède dans l'histoire soit mis en relation avec elle. Entre autres, la défaite à la fin de la Première Guerre Mondiale et ses suites contribuent aux tentatives d'explication de l'avènement au pouvoir des Nazis. En France, en revanche, cette guerre s'est terminée par une victoire. Au lieu « d'ouvrir » une page d'avenir, elle clôt une série d'événements et « répare " notamment la défaite de 1870-71.

En dehors de l'histoire elle-même et de différents courants de la didactique de l'histoire, une série de facteurs autres - qu'on a présentés de manière détaillée dans von Münchow 2013 - peut être invoquée pour expliquer les dissemblances constatées entre les manuels français et allemands: traditions historiographiques, cultures éducatives, contraintes éditoriales, etc. Pour opérer un retour sur le cadre théorique et méthodologique, on se contentera ici d'insister sur le fait que les représentations qui influent sur la construction du discours (que ce soit dans les manuels d'histoire ou ailleurs) - discours qui, à son tour, est constructeur de représentations - ne sont jamais univoques, ne seraient-ce que parce qu'elles sont toujours dues à plusieurs communautés parfois concurrentes. En l'occurrence, l'institution scolaire, les historiens, les didacticiens de l'histoire, les éditeurs scolaires, ou plus largement, la France et l'Allemagne, constituent un ensemble de groupes dont les valeurs et les croyances ne coïncident pas nécessairement.

Soulignons enfin l'importance de l'approche contrastive dans cette étude. La plupart des caractéristiques des manuels français ont en effet été relevées en quelque sorte "par défaut », du fait de l'absence de tel ou tel élément présent dans les manuels allemands. Il apparaît ici clairement que ces «absences » discursives constituent des clés pour la mise au jour de représentations tout aussi importantes que ce qui est clairement marqué dans les textes.

\section{BIBLIOGRAPHIE}

Authier-Revuz, Jacqueline. 2001. « Le discours rapporté », Tomassone, Roberte (éd.). Une langue :

le français, coll. Grands repères culturels (Paris : Hachette Éducation), 192-201

Authier-Revuz, Jacqueline. 2012. « Représentation du Discours Autre et catégorisation métalangagière », Grinshpun, Yana \& Judith Nyée-Doggen (éds). Regards croisés sur la langue française : usages, pratiques, histoire, Mélanges en l'honneur de Sonia Branca-Rosoff(Paris : Presses Sorbonne Nouvelle), 157-170

Baquès, Marie-Christine. 2007. «L'évolution des manuels d'histoire du lycée. Des années 1960 aux manuels actuels », Pédagogies de l'histoire 114, 121-149 
Bergmann, Klaus. 1979. « Multiperspektivität », Bergmann, Klaus et alii (éds). Handbuch der Geschichtsdidaktik, 2 vol. (Düsseldorf : Schwann), $1: 216-218$

Bergmann, Klaus. 2000. Multiperspektivität. Geschichte selber denken (Schwalbach : Wochenschau) Christophe, Barbara \& Kerstin Schwedes. 2015a. « Einleitung », Christophe, Barbara \& Kerstin Schwedes (éds). Schulbuch und Erster Weltkrieg. Kulturwissenschaftliche Analysen und geschichtsdidaktische Überlegungen (Göttingen : V\&R unipress), 7-13

Christophe, Barbara \& Kerstin Schwedes. 2015b. « Den Ersten Weltkrieg erzählen - Schulbücher als Erinnerungstexte lesen ", Christophe, Barbara \& Kerstin Schwedes (éds). Schulbuch und Erster Weltkrieg. Kulturwissenschaftliche Analysen und geschichtsdidaktische Überlegungen (Göttingen : V\&R unipress), 15-92

Christophe, Barbara \& Kerstin Schwedes (éds). 2015. Schulbuch und Erster Weltkrieg. Kulturwissenschaftliche Analysen und geschichtsdidaktische Überlegungen (Göttingen : V\&R unipress)

Confais, Jean-Paul. 2002. Temps mode aspect. Les approches des morphèmes verbaux et leurs problèmes à l'exemple du français et de l'allemand (Toulouse : PUM)

Dreesen, Philipp \& Joanna Judkowiak. 2011. « Passiv im Osten, kollektiv schuldig und selbstverständlich in Europa - Kritik an deutschen und polnischen Schulbüchern des Faches Geschichte mittels kontrastiver Diskurslinguistik », Aptum. Zeitschrift für Sprachkritik und Sprachkultur 7/1, 1-31. http://www.philippdreesen.de/Dreesen_Judkowiak_Passiv_im_Osten.pdf Dreesen, Philipp. 2015. « Sprache - Wissen - Kontingenz. Die Kontrastive Diskurslinguistik in der Schulbuchforschung am Beispiel deutscher und polnischer Geschichtsschulbücher », Kiesendahl, Jana \& Christine Ott (éds). Linguistik und Schulbuchforschung. Gegenstände - Methoden - Perspektiven (Göttingen : V\&R unipress), 53-83

Friedl, Sophie, Barbara Christophe \& Kerstin Schwedes. 2015. « Den Ersten Weltkrieg unterrichten - Didaktische Prinzipien und Herausforderungen im Spiegel von Schulbüchern », Christophe, Barbara \& Kerstin Schwedes (éds). Schulbuch und Erster Weltkrieg.

Kulturwissenschaftliche Analysen und geschichtsdidaktische Überlegungen (Göttingen : V\&R unipress), 93-154

Halbwachs, Maurice. 1997 [1950]. La mémoire collective (Paris : Albin Michel)

Lautier, Nicole \& Nicole Allieu-Mary. 2008. « La didactique de l'histoire », Revue française de pédagogie 162, 95-131

von Münchow, Patricia. 2009 [2004]. Les journaux télévisés français et allemands. Plaisir de voir ou devoir de s'informer (Paris : Presses Sorbonne Nouvelle)

von Münchow, Patricia. 2013. « Le discours sur les conflits entre nations dans les manuels d'histoire français et allemands. Représentations de la Première Guerre Mondiale ", Gonnot, Anne-Catherine, Nadine Rentel \& Stephanie Schwerter (éds). Dialogue(s) entre langues et cultures (Francfort-s.-Main : Peter Lang), 85-106

von Münchow, Patricia. 2016a. « Un enseignement tacite de l'Europe dans les manuels d'histoire français et allemands », Actes de la $12^{\mathrm{e}}$ Journée Pierre Guibbert du 3 février 2016 « L'Europe et les Européens dans les manuels scolaires », Université de Montpellier 3. http://www.fde.univmontp2.fr/internet/site/cedrhe/_img_cedrhe/jepg/jepg_id_49.pdf

von Münchow, Patricia. 2016b. « Discours rapporté, rapport à l'histoire et construction de communautés : la Première Guerre Mondiale dans les manuels scolaires d'histoire français et allemand », Barthelmebs-Raguin, Hélène et alii (éds.) Le discours rapporté... une question de temps (Paris : Classiques Garnier), à paraître. 
Ott, Christine. 2015. «Bildungsmedien als Gegenstand linguistischer Forschung. Thesen, Methoden, Perspektiven », Kiesendahl, Jana \& Christine Ott (éds). Linguistik und Schulbuchforschung. Gegenstände - Methoden - Perspektiven (Göttingen : V\&R unipress), 19-37

Pandel, Hans-Jürgen. 2005. Geschichtsunterricht nach Pisa. Kompetenzen, Bildungsstandards und Kerncurricula (Schwalbach : Wochenschau Verlag)

Rebotier, Aude. 2010. «Les emplois stéréotypiques des temps narratifs en français ", Revue Interdisciplinaire Textes \& contextes 5, « Stéréotypes en langue et en discours ». http://revuesshs.ubourgogne.fr/textes\&contextes/document.php?id=1256 ISSN 1961-991X

Weinrich, Harald. 1985 [1964]. Tempus. Besprochene und erzählte Welt (Stuttgart \& Berlin \& Cologne \& Mayence : Kohlhammer)

Winter, Jay \& Antoine Prost. 2005. The Great War in History. Debates and Controversies, 1914 to Present (Cambridge : Cambridge University Press)

\section{NOTES}

1. Il s'agit là d'une discipline très proche de celle que j'ai moi-même appelée d'abord « linguistique du discours comparative » (von Münchow 2009 [2004]), puis " analyse du discours contrastive », mais dont les auteurs ne s'inspirent pas.

2. « Erinnerungskulturen [...] werden in Schulbüchern wie in einem Brennglas gebündelt. »

3. Pour une description détaillée de cette approche, voir notamment von Münchow 2009 [2004].

4. Figurent entre crochets les termes par lesquels on renverra infra aux manuels en question.

5. Les caractères gras ainsi que les italiques et les soulignements dans les extraits cités au cours de ce texte figurent comme tels dans les manuels.

6. En modalisation autonymique d'emprunt (von Münchow 2009 [2004] : 99-101), qui fait partie des types de base du discours rapporté, le discours autre est «ce par quoi est opérée une modalisation" (Authier-Revuz 2001: 195) de l'énoncé rapportant. La représentation implique non seulement le contenu, mais aussi le plan de l'expression, à travers des mots « dont il est fait "mention", via de l'autonymie renvoyant aux mots eux-mêmes » (ibid.).

7. La formule étant mieux connue en France dans sa version originale qu'en traduction française, j'ai conservé l'original allemand ici.

8. L'une des sources figurant dans cette section est le discours d'August Bebel, président du SPD, au Reichstag (1911) contre la course à l'armement (Entdecken und Verstehen : 143).

9. C'est ce que concluent aussi Winter et Prost (2005).

\section{RÉSUMÉS}

Cet article fait état des résultats d'une analyse du discours contrastive de manuels d'histoire en France et en Allemagne, et plus précisément des chapitres portant sur la Première Guerre Mondiale. Il s'agit de montrer quelles représentations les auteurs des manuels étudiés proposent du rapport entre « le passé » et le présent dans lequel a lieu l'enseignement/apprentissage. Le positionnement dans le temps au sens large est étudié à travers les titres des manuels, l'emploi des temps grammaticaux et d'autres marqueurs temporels, mais aussi par l'intermédiaire de 
différents types de descriptions, du discours rapporté, des comparaisons d'événements et du positionnement de l'élève dans les tâches à accomplir. On constate qu'une perspective globalement contemporaine à la publication dans les manuels français, mais que les auteurs ne parviennent pas à respecter systématiquement, s'oppose à un constant aller-retour conscient et didactisé entre passé et présent dans les manuels allemands.

This paper presents the results of a cross-cultural discourse analysis of the chapters on Word War I in French and German history textbooks. More precisely, the study deals with textbook authors' representations of the relationship between "the past" and the present of the teaching and learning situation. It shows that authors convey - and construct - these representations by a variety of linguistic and discursive means such as the title of their textbook, temporal markers, different types of description and of reported speech, as well as comparisons of events to other events and the positioning of the students in the tasks they are asked to accomplish. In general, French textbook authors take on a contemporary perspective, but do not always succeed in rigorously following it, whereas German authors go back and forth between the past and the present time and engage the students in doing so as well.

INDEX

Mots-clés : analyse du discours contrastive, manuels d'histoire, Première Guerre Mondiale Keywords : cross-cultural discourse analysis, history textbooks, World War I

\section{AUTEUR}

\section{PATRICIA VON MÜNCHOW}

Université Paris Descartes - Sorbonne Paris Cité, Laboratoire EDA 